Gynäkologische Endokrinologie 2017 · 15:259 https://doi.org/10.1007/s10304-017-0160-0

Online publiziert: 4. Oktober 2017

(c) Springer Medizin Verlag GmbH 2017

CrossMark

\section{G. Griesinger}

Sektion für Gynäkologische Endokrinologie und Reproduktionsmedizin, Klinik für Frauenheilkunde und Geburtshilfe, Universitätsklinikum Schleswig-Holstein, Campus Lübeck, Lübeck, Deutschland

\title{
Kinderwunsch - Operation Ja oder Nein?
}

Die Reproduktionschirurgie der Frau kann als eine Subspezialität der minimal-invasiven Chirurgie in der Gynäkologie aufgefasst werden. Das Ziel der Reproduktionschirurgie ist die Integration der chirurgischen Behandlung und einer umfassenden ärztlichen Betreuung unter Berücksichtigung endokriner, geburtshilflicher, sexualmedizinischer und sozialer Aspekte von Frauen mit Kinderwunsch. Nur die Kenntnis aller fertilitätsmedizinisch relevanten Aspekte und Techniken erlaubt eine individuelle Planung des operativen Vorgehens, welches immer die Implikationen auf die Fertilität berücksichtigen muss. Von besonderer Bedeutung sind dabei endokrine und nichtendokrine Begleit- und Folgebehandlungen sowie Aspekte von Schwangerschaftsrisiken und Entbindungsmodus.

\section{》) Die Anwendungsmöglich- keiten der minimal-invasiven Chirurgie haben sich gewandelt}

In den vergangenen Jahren haben sich die Anwendungsmöglichkeiten der minimal-invasiven Chirurgie im Besonderen durch neue Techniken auch aus anderen Spezialgebieten gewandelt. Eine holistische Betrachtung von Problemstellungen wie beispielsweise polyzystisches Ovarsyndrom, Adhäsionssitus, Leiomyome oder Endometriose wird von unseren Patienten zu Recht erwartet. Vor diesem Hintergrund ist die vorrangige Durchführung der In-vitro-Fertilisation in rein ambulant tätigen ärztlichen Praxen notwendigerweise durch Vernetzung mit entsprechend geschulten und erfahrenen mikrochirurgischen Abteilungen zu gewährleisten. Wünschenswert ist aber, dass auch in Zukunft eine hinlängliche Zahl an Reproduktionsmedizinern mit Kenntnissen und Fertigkeiten der Reproduktionschirurgie vertraut gemacht werden kann, wie dies auch die Weiterbildungsordnung des Schwerpunkts gynäkologische Endokrinologie und Reproduktionsmedizin vorsieht.

Das vorliegende Heft widmet sich ausgewählten, für die Reproduktionsmedizin besonders wichtigen Krankheitsbildern, für deren Abhandlung wir jeweils ausgewiesene Experten gewinnen konnten. Einen besonderen Stellenwert messen wir der Reproduktionschirurgie im Kontext des weiblichen Fertilitätserhalts bei. Die Methode der Kortexentnahme und Retransplantation darf inzwischen als etabliertes Verfahren betrachtet werden. Die Verschränkung von geweberechtlichen Anforderungen an die Entnahme, Aufbereitung und Verarbeitung von Geweben mit operativer Expertise und Erfahrung wird absehbar zur Herausbildung einiger weniger Kompetenzzentren in Deutschland führen.

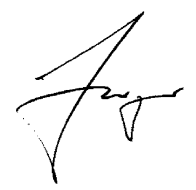

G. Griesinger

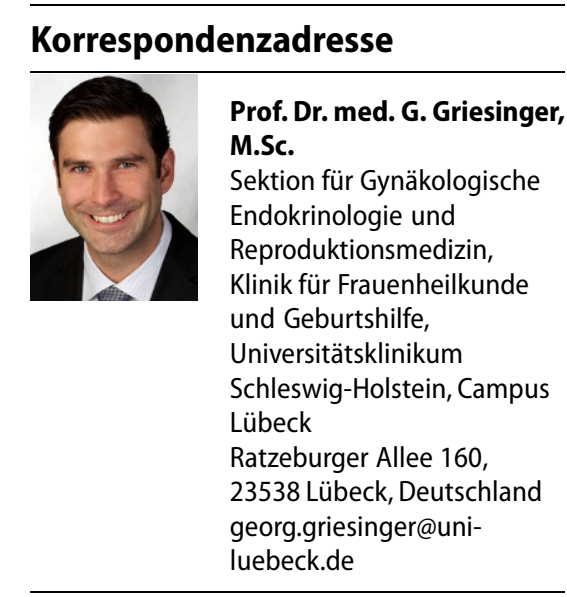

Interessenkonflikt. G. Griesinger gibt an, dass kein Interessenkonflikt besteht. 